\title{
ESTUDO RETROSPECTIVO DE MIÍASES EM CÃES ATENDIDOS NO HOSPITAL VETERINÁRIO DA UNIVERSIDADE FEDERAL DO PIAUÍ
}

\author{
Francisco Michael Junior Costa ${ }^{1}$; Dayana Maria do Nascimento²; Marcus Valérius de \\ Matos Freitas ${ }^{3}$; Marcelo Campos Rodrigues ${ }^{4}$; Luanna Soares de Melo Evangelista ${ }^{5}$ \\ ${ }^{1}$ Acadêmico de Medicina Veterinária, Centro de Ciências Agrárias, Universidade \\ Federal do Piauí \\ ${ }^{2}$ Residente em Clínica e Cirurgia de Pequenos Animais, Centro de Ciências Agrárias, \\ Universidade Federal do Piauí \\ ${ }^{3}$ Médico Veterinário, Hospital Veterinário Universitário, Centro de Ciências Agrárias, \\ Universidade Federal do Piauí \\ ${ }^{4}$ Prof. Dr. Departamento de Clínica e Cirurgia Veterinária, Centro de Ciências \\ Agrárias, Universidade Federal do Piauí \\ ${ }^{5} \mathrm{Prof}^{\mathrm{a}} \mathrm{Dr}^{\mathrm{a}}$ Departamento de Parasitologia e Microbiologia, Centro de Ciências da \\ Saúde, Universidade Federal do Piauí (luannaufpi@gmail.com)
}

Recebido em: 06/04/2018 - Aprovado em: 10/06/2018 - Publicado em: 20/06/2018 DOI: 10.18677/EnciBio_2018A48

\begin{abstract}
RESUMO
Miíases são enfermidades parasitárias provocadas pela infestação de larvas de moscas que se alimentam de tecido vivo ou morto de humanos e animais. Essas infestações podem ocorrer em diversas áreas do corpo, constituindo-se um sério problema sanitário e econômico. As larvas da mosca Cochliomyia hominivorax são os parasitos mais relacionados com essa doença, podendo ser encontradas com grande frequência no Brasil. O objetivo desta pesquisa foi realizar um levantamento epidemiológico da casuística de cães com miíases atendidos no Hospital Veterinário da Universidade Federal do Piauí (HVU/UFPI), no período de 2015 a 2017. Este levantamento foi obtido por meio das fichas de atendimento clínico arquivadas no HVU/UFPI de 175 cães com diagnóstico da doença. Foram obtidos dados quanto ao sexo, idade, raça e localização das miíases. Os resultados apontaram que cães machos apresentaram uma maior prevalência dessa enfermidade, representando $65.7 \%$ dos casos atendidos. A maioria dos animais comprometidos eram adultos (46.9\% dos casos), sendo que os cães sem raça definida (SRD) foram os mais acometidos, com $58.9 \%$, seguidos da raça Pastor Alemão (17.2\%) e a cavidade auditiva $(25.7 \%)$ ficou entre as regiões anatômicas mais afetadas pelos parasitos. Conclui-se que a casuística de cães com miíases atendidos no HVU/UFPI foi relativamente alta durante o período do estudo, sendo importante que os tutores de cães sejam orientados quanto às medidas de prevenção e controle dessa parasitose em seus animais.
\end{abstract}

PALAVRAS-CHAVE: cães, casuística, epidemiologia, miíases. 


\title{
RETROSPECTIVE STUDY OF MYIASIS IN DOGS ATTENDED AT THE VETERINARY HOSPITAL OF THE FEDERAL UNIVERSITY OF PIAUÍ
}

\begin{abstract}
Myiasis are parasitic diseases caused by infestation of fly larvae that feed on living or dead tissue of humans and animals These infestations can occur in several areas of the body, constituting a serious health and economic problem. The larvae of the Cochliomyia hominivorax fly are the parasites most related to this disease, which can be found frequently in Brazil. The aim of this research was to carry out an epidemiological survey of the casuistry of dogs with myiasis attended at the Veterinary Hospital of the Federal University of Piauí (HVU/UFPI), from 2015 to 2017. This survey was obtained through the clinical records of 175 dogs with diagnosis of the disease. Data on sex, age, race and location of myiasis were obtained. The results showed that male dogs presented a higher prevalence of this disease, representing $65.7 \%$ of the cases attended. Most of the animals involved were adults (46.9\% of the cases), mixed breed dogs were the most affected, with $58.9 \%$, followed by the German Shepherd breed $(17.2 \%)$ and the auditory cavity $(25.7 \%)$ was among the anatomical regions most affected by the parasites. It is concluded that the casuistry of dogs with myiasis attended at HVU/UFPI was relatively high during the study period, and it is important that dog tutors are advised on the prevention and control of this parasitosis in their animals.
\end{abstract}

KEYWORDS: dogs, casuistry, epidemiology, myiasis.

\section{INTRODUÇÃO}

Miíase é a infestação de vertebrados, humanos ou animais, com larvas de dípteros que, por determinado período de tempo, se alimentam de tecidos vivos ou mortos de seus hospedeiros, além de suas substâncias corporais (MONTEIRO, 2014). É geralmente causada por moscas da família Calliphoridae, e a Cochliomyia hominivorax é o agente etiológico mais frequentemente relacionado a esta enfermidade (OLEA et al., 2014).

É um dos principais problemas parasitários em animais de companhia (cães e gatos); animais de produção (principalmente ruminantes e suínos) e silvestres. Também pode ocorrer em humanos, principalmente em pessoas debilitadas, idosas ou com deficiência mental (FARES et al., 2005), gerando impactos sanitários e econômicos nas populações acometidas.

A classificação desta doença é baseada na sua localização no corpo do hospedeiro, podendo ser cutânea (por depósito de ovos de moscas em ulcerações de pele), cavitária (encontradas nas cavidades nasal, oral, anal, auditiva, orbital, etc.) e intestinal (por ingestão de larvas de moscas em alimentos contaminados) (MCGRAW; TURIANSKY, 2008) ou em termos etológicos, podem ser classificadas como obrigatória, facultativa e pseudomiíase (GOMEZ et al., 2003; MONTEIRO, 2014).

Em cães infestados por larvas de moscas ocorre destruição dos tecidos e muitas vezes geram lesões bastante extensas. A gravidade desta parasitose está diretamente relacionada à localização da lesão no corpo do hospedeiro e à quantidade de larvas presentes no tecido (CARDOZO; RAMANDINHA, 2007).

Nestes animais, as miíases, em sua grande maioria, estão associadas à negligência do tutor em relação ao tratamento de feridas cutâneas ou na falta de observação quanto ao acúmulo de fezes e urina nos pelos dos animais, o que pode atrair moscas para a oviposição ou larviposição (ANDERSON; HUITSON, 2004; 
COOPER; COOPER, 2007), afetando, com maior frequência, cães de áreas urbanas, adultos machos e com pelagem longa e escura (CRAMER-RIBEIRO et al., 2003).

Diante da incidência e importância dessa enfermidade parasitária na população canina, esta pesquisa teve como objetivo realizar um levantamento epidemiológico da casuística de cães com miíases atendidos no Hospital Veterinário da Universidade Federal do Piauí (HVU/UFPI), no período de 2015 a 2017.

\section{MATERIAL E MÉTODOS}

Este trabalho é do tipo retrospectivo com coleta de fontes secundárias sobre a casuística de cães com diagnóstico de miíases que realizaram atendimento clínico no HVU/UFPI, município de Teresina, PI, durante o período de 01 de janeiro de 2015 a 31 de julho de 2017. As fichas foram preenchidas no momento de cada consulta e todo o procedimento adotado foi realizado pelo Médico Veterinário responsável pelo atendimento clínico. Essas fichas foram arquivadas no sistema computadorizado do HVU/UFPI.

A coleta e avaliação dos dados foram realizadas entre os meses de julho e novembro de 2017 por meio de acesso ao sistema do HVU/UFPI, com autorização prévia da Direção do hospital. As informações dos dados de interesse foram colhidas nas fichas de atendimento de casos clínicos confirmados de miíases em cães.

Foram obtidas informações quanto ao sexo dos animais (se machos ou fêmeas), quanto a idade (de um mês a dezoito anos), sendo agrupados por categoria animal: jovens, menores de 01 ano; adultos, de 01 a 08 anos e idosos, acima dessa idade. Também foram avaliados parâmetros quanto a raça, localização corporal da miíase (se em cavidade auditiva, órbita ocular, nasal, oral, região perianal, genital, tronco, membros anteriores e posteriores ou região não definida) e por zona domiciliar do animal (zona leste, sul, sudeste, norte, centro, zona rural do município de Teresina ou outras cidades circunvizinhas). Todos os resultados foram organizados em forma de tabelas, contendo o " $n$ " e a porcentagem para cada parâmetro avaliado.

\section{RESULTADOS E DISCUSSÃO}

De acordo com os registros arquivados nas fichas de atendimento clínico dos animais com miíases do HVU/UFPI, foram contabilizados 175 cães acometidos com a doença, sendo a maioria cães machos, com $65.7 \%$ dos casos, como mostra a tabela 01.

TABELA 01. Frequência de cães acometidos por miíases atendidos no HVU/UFPI, Teresina, PI, 2015-2017, de acordo com o sexo.

\begin{tabular}{lcc}
\hline Sexo & $\mathrm{N}^{\circ}$ & Frequência (\%) \\
\hline Machos & 115 & 65.7 \\
Fêmeas & 060 & 34.3 \\
Total & 175 & 100 \\
\hline
\end{tabular}

Por meio deste estudo foi possível observar que essa enfermidade parasitária foi um achado clínico de grande ocorrência em cães atendidos no HVU/UFPI, durante o período avaliado. Essa alta prevalência pode estar relacionada ao descaso dos tutores frente a presença de ferimentos em seus animais e ausência de tratamento em tempo hábil, uma vez que a instalação dos ovos e desenvolvimento 
das larvas de moscas causadoras de miíases, principalmente da Cochliomyia hominivorax, necessitam de um período médio de cinco dias para se desenvolverem e, normalmente, se alimentam de alguma lesão no tecido (MONTEIRO, 2014). O ciclo de vida dessa espécie revela que as moscas adultas colocam de 200 a 300 ovos nas bordas das feridas, e estas, uma vez infectadas, liberam um odor característico que estimulam outras moscas a ovipor (ROSSI et al., 2002; OLEA et al., 2014), aumentando, por sua vez, o número de larvas em cada lesão. Outros trabalhos também relataram um grande número de animais com diagnóstico de miíases (SCHNUR et al., 2009).

Neste estudo, cães machos foram os mais acometidos pela enfermidade, fato que pode ser justificado pelo comportamento canino, uma vez que estes mantêm hábitos de marcação de território e quando estão à procura de fêmeas, se envolvem em brigas com outros cães (ROSSI, 2008), podendo provocar ferimentos que facilitam a deposição de ovos e larvas de moscas causadoras de miíases.

Trinta e quatro cães tinham menos de um ano de idade, sendo considerados animais jovens e 141 eram animais maiores de um ano, inclusive, destes, 59 tinham mais de oito anos de idade, ou seja, foram classificados como cães idosos. A tabela 02 mostra esses resultados.

TABELA 02. Frequência de cães acometidos por miíases atendidos no HVU/UFPI, Teresina, PI, 2015-2017, conforme a idade.

\begin{tabular}{lll}
\hline Idade & $\mathrm{N}^{\circ}$ & Frequência $(\%)$ \\
\hline Jovens ( $\leq 01$ ano) & 34 & 19.4 \\
Adultos (Entre 01-08) & 82 & 46.9 \\
Idosos ( $\geq 08$ anos) & 59 & 33.7 \\
Total & 175 & 100 \\
\hline
\end{tabular}

Cães adultos têm mais acesso à rua e com isso estão menos protegidos, o que favorece a contaminação por miíases. Esses resultados corroboram com outros autores que também revelaram cães adultos e idosos como os mais acometidos por esta enfermidade (CRAMER-RIBEIRO et al., 2003). Na tabela 03 é possível observar algumas raças de cães parasitados por miíases, sendo os sem raça definida (SRD) na maioria dos casos avaliados.

TABELA 03. Frequência de cães acometidos por miíases atendidos no HVU/UFPI, Teresina, PI, 2015-2017, segundo a raça.

\begin{tabular}{lll}
\hline Raças & $N^{\circ}$ & Frequência \\
\hline SRD & 103 & 58.9 \\
Pastor Alemão & 30 & 17.2 \\
Poodle & 13 & 7.4 \\
Pit Bull & 06 & 3.4 \\
Outras Raças & 23 & 13.1 \\
Total & 175 & 100 \\
\hline
\end{tabular}

A incidência de cães SRD (58.9\%) acometidos por miíases foi a maior entre as raças avaliadas neste trabalho, fato esse que pode ser justificado, em geral, por pertencerem a tutores de baixa renda que, muitas vezes, negligenciam seus animais devido às suas condições financeiras ou, simplesmente, por falta de cuidados básicos com a saúde de seus cães (SCOTT et al., 1996; CRAMER-RIBEIRO et al., 2003), onde a maioria são criados soltos em quintais abertos, com higienização precária, tendo fácil acesso a locais com lixos e moscas. 
Os resultados encontrados nesse trabalho revelaram um número considerado de cães da raça Pastor Alemão diagnosticados com essa enfermidade. $O$ fato de eles serem cães de grande porte, normalmente são mantidos fora das residências para guarda da casa, o que facilita a exposição às moscas e, consequentemente, às miísaes, além do que sua pelagem longa também favorece essa infestação (CRAMER-RIBEIRO et al., 2003; ANDERSON; HUITSON, 2004).

A localização corporal das miíases nos cães deste estudo foi registrada de acordo com a região anatômica de cada animal, conforme mostra a tabela 04.

TABELA 04. Frequência de cães acometidos por miíases atendidos no HVU/UFPI, Teresina, PI, 2015-2017, de acordo com a localização corporal nos animais.

\begin{tabular}{lll}
\hline Localização & $\mathrm{N}^{\circ}$ & Frequência (\%) \\
\hline Cavidade auditiva & 45 & 25.7 \\
Tronco & 22 & 12.6 \\
Membros Anteriores & 19 & 10.9 \\
Membros Posteriores & 13 & 7.4 \\
Ocular & 12 & 6.9 \\
Genital & 11 & 6.3 \\
Perianal & 10 & 5.7 \\
Nasal & 07 & 4.0 \\
Oral & 06 & 3.4 \\
Não relatada & 30 & 17.1 \\
Total & 175 & 100 \\
\hline
\end{tabular}

Houve uma predileção das larvas de moscas pelas cavidades auditivas dos cães parasitados (25.7\% dos casos), e na maioria destes animais também foi observada otite. Pastor Alemão e poodle são cães que apresentam essa moléstia frequentemente, podendo, inclusive, ter como consequência os casos de miíases nas orelhas (CRAMER-RIBEIRO et al., 2003), o que possivelmente ocorreu neste trabalho. Portanto, a prevenção da otite também pode ajudar a evitar a miíase.

Trabalhos relataram o envolvimento humano com larvas de moscas, comprometendo as cavidades nasais, auditivas e ocular de um paciente de origem rural que foi internado em estado grave para tratamento (RODRÍGUEZ-DIEGO et al., 2007; DIEGO et al., 2016). Outras localizações também já foram descritas na literatura tanto em animais como em humanos, sendo o tronco também um órgão de predileção das larvas (FARES et al., 2005).

Muitas localizações anatômicas das miíases nos animais deste trabalho (17.1\%) não foram anotadas nas fichas de atendimento clínico do HVU/UFPI, isso mostra, inclusive, uma falha no padrão de organização do preenchimento das fichas daquele estabelecimento veterinário, devendo ser reavaliado criteriosamente esse tipo de informação nas futuras avaliações. Observou-se que a zona leste da capital piauiense foi a região que teve a maior incidência de cães com miíases, seguida da zona sul (tabela 05). 
TABELA 05. Frequência de cães acometidos por miíases atendidos no HVU/UFPI, Teresina, PI, 2015-2017, segundo a zona domiciliar.

\begin{tabular}{lll}
\hline Regiões & $\mathrm{N}^{\circ}$ & Frequência (\%) \\
\hline Leste & 52 & 29.7 \\
Sul & 49 & 28.0 \\
Norte & 41 & 23.5 \\
Sudeste & 15 & 8.5 \\
Zona rural & 09 & 5.1 \\
Centro & 05 & 2.9 \\
Outras cidades & 04 & 2.3 \\
Total & 175 & 100 \\
\hline
\end{tabular}

Tal achado pode ser justificado pelo fato do HVU/UFPI estar localizado na zona leste do município em questão, mantendo uma grande demanda de atendimento de cães e gatos. Os tutores dos animais possivelmente procuram o hospital pelo custo mais acessível quando comparado com outras clínicas veterinárias, bem como por se tratar de um hospital-escola, onde oferece diversos tipos de serviços na área da Medicina Veterinária em um único ambiente, facilitando, assim, a vida destes tutores, que, na maioria das vezes, já tentaram algum tipo de tratamento empírico em casa, sem solução e, com isso, buscam atendimento tardio para seus animais desejando resolver o caso.

\section{CONCLUSÃO}

Conclui-se que a casuística de cães com miíases atendidos no HVU/UFPI foi relativamente alta durante o período do estudo, sendo importante que os tutores de cães sejam orientados quanto às medidas de prevenção e controle, a fim de diminuir o número de casos dessa parasitose na população canina do município de Teresina, Piauí.

\section{AGRADECIMENTOS}

Ao Diretor do Hospital Veterinário da Universidade Federal do Piauí, Prof. Dr. João Macedo de Sousa, por autorizar a busca dos resultados para este trabalho.

\section{REFERÊNCIAS}

ANDERSON, G. S.; HUITSON, N. R. Myiasis in pet animals in British Columbia: the potential of forensic entomology for determining duration of possible neglect. The Canadian Veterinary Journal, v. 45, n. 12, p. 993-998, 2004. Disponível em: <https://www.ncbi.nlm.nih.gov/pmc/articles/PMC554754/>.

CARDOZO, S. V.; RAMADINHA, R. R. Avaliação do tratamento de miíases em cães através da utilização do nitenpyram. Revista Brasileira de Ciência Veterinária, v. 14, n. 3, p. 139-142, 2007. Disponível em: <http://www.rbcv.uff.br/rbcv/article/viewFile/433/729>.

CRAMER-RIBEIRO, B. C.; SANAVRIA, A.; OLIVEIRA, M. Q.; SOUZA, F. S. Inquiry of cases of myiasis by Cochliomyia hominivorax in dogs (Canis familiaris) of the Northern and Western zones of Rio de Janeiro city in 2000. Brazilian Journal of Veterinary Research and Animal Science, v. 40, n. 1, p. 13-20, 2003. Disponível em: $<$ http://www.scielo.br/scielo.php?script=sci_arttext\&pid=S1413- 
COOPER, J. E.; COOPER, M. E. Introduction to Veterinary and Comparative Forensic Medicine. Blackwell Publishing: Oxford, 2007. 415p.

DIEGO, J. G. R.; OROZCO, J. L. O.; CASTILLEJA, Y. S.; GARCIA, J. A. EI Gusano Barrenador del Ganado, Cochliomyia hominivorax (Diptera:Calliphoridae): un problema en la salud animal y humana. Revista de Salud Animal, v. 38, n. 2, p. 120-130, 2016. Disponível em: <http://scielo.sld.cu/scielo.php? script=sci_arttext\&pid=S0253-570X2016000200008>.

FARES, N. H.; MELO, D. V.; STUCCHI, N.; CARVALHOSA, A. A.; CASTRO, P. H. S.; SIQUEIRA, C. R. B. Miíase em paciente com 10 anos de idade: Relato de caso clínico e revisão de literatura. Revista de Clínica e Pesquisa Odontológica, v. 1, n. 4, p. 49-54, 2005. 4 Disponível <https://www.researchgate.net/publication/33550752_MIIASE_EM_PACIENTE_COM 10_ANOS_DE_IDADE_RELATO_DE_CASO_CLINICO_E_REVISAO_DA_LITERA TURA $>$.

GOMEZ, R. S., PERDIGÃO, P. F., PIMENTA, F. J. G. S., RIOS LEITE, A. C., TANOS DE LACERDA, J. C., CUSTÓDIO NETO, A. L. Oral myiasis by screwworm Cochliomyia hominivorax. International Journal of Oral \& Maxillofacial Surgery, v. 41, n. 2, p. 115-116, 2003. Disponível em: <https://www.ncbi.nlm.nih.gov/pubmed/12694705>.

MCGRAW, T. A.; TURIANSKY, G. W. Cutaneos myiasis. Journal of American Academy of Dermatology, v. 58, n. 6, p. 907-926, 2008. Disponível em: <https://www.ncbi.nlm.nih.gov/pubmed/18485982>.

MONTEIRO, S. G. Parasitologia na Medicina Veterinária. São Paulo: Roca, 2014. $356 \mathrm{p}$.

OLEA, M. S..; CENTENO, N.; AYBAR, C. A. V.; ORTEGA, E. S.; GALANTE, G. B.; OLEA, L.; JURI, M. J. D. First Report of Myiasis Caused by Cochliomyia hominivorax (Diptera: Calliphoridae) in a Diabetic Foot Ulcer Patient in Argentina. Korean Journal of Parasitology, v. 52, n. 1, p. 89-92, 2014. Disponível em: <https://www.ncbi.nlm.nih.gov/pubmed/24623889>.

RODRÍGUEZ-DIEGO, J. G.; CÓRDOVA-RAMOS, G.; AROZARENA, R. First notification of cattle screw worm (Cochliomyia hominivorax) in a human case, in Cuba. Revista de Salud Animal, v. 29, n. 3, p. 193, 2007. Disponível em: <http://scielo.sld.cu/scielo.php?script=sci_arttext\&pid=S0253-570X2007000300010>.

ROSSI, G. C.; MARILUIS, J. C.; SCHNACK, J. A.; SPINELLI, G. R. Dípteros vectores (Culicidae y Calliphoridae) de la provincia de Buenos Aires. ProBiota, v. 3, n. $4,2002.45$. 20 Disponível <http://sedici.unlp.edu.ar/bitstream/handle/10915/15832/Revista_completa.pdf? sequence $=1>$.

ROSI, A. Comportamento canino - como entender, interpretar e influenciar o comportamento dos cães. Revista Brasileira de Zootecnia, v. 37, n. spe, 2008. 
Disponível em: <http://www.scielo.br/scielo.php?script=sci_arttext\&pid=S151635982008001300007>.

SCHNUR, H. J.; ZIVOTOFSKY, D.; WILAMOWSKI, A. Myiasis in domestic animals in Israel. Veterinary Parasitology, v. 161, n. 3-4, p. 352-355, 2009. Disponível em: <https://www.sciencedirect.com/science/article/pii/S0304401709000788>.

SCOTT, D. W.; MILLER, W. H.; GRIFFIN, C. E. Doenças parasitárias da pele. In: SCOTT, D. W.; MILLER, W. H.; GRIFFIN, C. E. Muller \& Kirk, Dermatologia de pequenos animais. Philadelphia: W. B. Saunders Company,. p. 423-427.1996. 